\title{
R1507, A Fully Human Monoclonal Antibody Targeting IGF-1R, Is Effective Alone and in Combination With Rapamycin in Inhibiting Growth of Osteosarcoma Xenografts
}

\author{
E. Anders Kolb, MD, ${ }^{1 *}$ Davida Kamara, ${ }^{1}$ Wendong Zhang, ${ }^{2}$ Juan Lin, ${ }^{3}$ Pooja Hingorani, $\mathrm{MD}_{,}{ }^{4}$ \\ Laurence Baker, $\mathrm{MD}^{5}$ Peter Houghton, $\mathrm{PhD},{ }^{6}$ and Richard Gorlick, $\mathrm{MD}^{2}$
}

\begin{abstract}
Background. The combination of rapamycin and R1507, a fully human IgG1 monoclonal antibody targeting the IGF-1 receptor (IGF$1 \mathrm{R})$, in osteosarcoma xenograft tumors in vivo is evaluated in this report. Procedure. Six osteosarcoma xenograft tumor models were evaluated for growth inhibition after monotherapy with R1507, rapamycin, and the combination of both drugs. Phosphorylation of proteins involved in IGF-1R signaling is evaluated at various time points by immunoblotting. Results. IGF-1R was expressed in five of the six human osteosarcoma tumor lines. Objective responses to R1507 were seen in four of the six tumor lines (OS1, OS2, OS9, and OS17) including one complete response in OS1. IGF-1R protein levels did not predict degree of response to R1507 in the sensitive tumors. However, in one of the two R1507-resistant tumors (OS33), there was a minimal expression of IGF-1R. An increase in AKT
\end{abstract}

phosphorylation was observed in all the osteosarcoma tumors treated with rapamycin. However, phosphorylation of AKT was inhibited when rapamycin was used in combination with R1507. In three of the xenograft tumor lines, there was an improvement in response when R1507 was used in combination with rapamycin. Conclusions. IGF-1R inhibition by R1507 induced tumor growth delays and improvement in event-free survival in four of six osteosarcoma xenograft tumor lines. R1507 negates increased signaling through AKT in response to mammalian target of rapamycin inhibition, suggesting that the combination is worthy of further evaluation in patients. As R1507 and other IGF-1R inhibitors advance in clinical trials, it will be important to understand biomarkers of response and pathways of resistance. Pediatr Blood Cancer 2010;55:67-75. (c) 2010 Wiley-Liss, Inc.

Key words: IGF-1R; pediatric sarcomas; preclinical testing; rapamycin

\section{INTRODUCTION}

Osteosarcoma is the most common primary malignant bone tumor in children and young adults. The overall survival rate remains around $60-70 \%$. Survival rates in patients with pulmonary metastases are generally less than $30 \%$. Approximately, one-third of patients with only local disease at diagnosis will relapse with pulmonary metastases [1]. Over the last 20 years, there have been few changes in standard chemotherapy treatment regimens for patients with osteosarcoma.

In normal bone, insulin-like growth factor (IGF) signaling is involved in osteogenesis and bone homeostasis [2]. While there is no conclusive evidence published to date to implicate IGF-1 and/or IGF-2 in autocrine growth in osteosarcoma, consistent expression of IGF-1R, IGF-1, and IGF-2 has been reported in osteosarcoma cell lines and patient samples [3]. Evidence of IGF-mediated growth in osteosarcoma can be inferred from experiments demonstrating inhibition of growth and metastases of murine osteosarcoma following hypophysectomy [4], and inhibition of tumor growth in xenografts following treatment with anti-IGF-1R antibody. Additionally, an increased risk of osteosarcoma is reported among patients with a single nucleotide polymorphism in IGF2R suggesting a possible role for this variant IGF-2R in the pathogenesis of osteosarcoma [5].

IGF-1R is capable of binding both IGF-1 and IGF-2, leading to autophosphorylation of the receptor. When IGF-1R is phosphorylated, insulin receptor substrates 1-4 (IRS1-4) and the Src homology collagen-like adaptor protein (Shc) can initiate intracellular signaling cascades. Phosphorylated IRS-1 activates the p85 regulatory subunit of phosphatidylinositol 3-kinase (PI3K). When the p85 and p1 10 subunits of PI3K bind to IRS, phosphatidylinositol-4,5-biphoshate is converted to phosphatidylinositol-3,4,5biphoshate $\left(\mathrm{PIP}_{3}\right)$. Phosphoinositide-dependent kinase-1 is then activated by $\mathrm{PIP}_{3}$, resulting in phosphorylation and activation of the serine/threonine kinase AKT. Substrates responsible for the diverse functions of AKT include glycogen synthase kinase-3, involved in enhancing intracellular metabolism; the mammalian target of rapamycin (mTOR), involved in protein synthesis and cell growth; BAD (a Bcl-2 family member), involved in cell survival; and $\mathrm{p} 28^{\mathrm{Kip} 1}$, involved in cell proliferation. Regulation and inhibition of PI3K signaling may be mediated in part by S6K, which when activated by mTOR, phosphorylates the serine residues on IRS-1, thus marking it for degradation [6,7]. In parallel to the signaling activated though PI-3K, Shc activates Ras. Once activated, Ras initiates signaling through Raf, which ultimately leads to activation and phosphorylation of the mitogen-activated protein kinase- 1 and -2 (MAPK) through the MAPK/ERK kinases (MEK1 and MEK2) [6]. Activation of MAPK through Ras results in enhanced proliferation, migration, and survival [6,7].

It has been known for 20 years that IGF signaling is important to the growth and survival of sarcomas [8]. The recent development of

${ }^{1}$ Division of Hematology and Oncology, Alfred I. duPont Hospital for Children, Nemours Center for Childhood Cancer Research, Wilmington, Delaware; ${ }^{2}$ Department of Pediatric Hematology/ Oncology, Albert Einstein College of Medicine, Bronx, New York; ${ }^{3}$ Division of Biostatistics, Department of Epidemiology and Population Health, Albert Einstein College of Medicine, Bronx, New York; ${ }^{4}$ Division of Hematology and Oncology, Phoenix Children's Hospital, Phoenix, Arizona; ${ }^{5}$ Division of Hematology and Oncology, University of Michigan Comprehensive Cancer Center, Ann Arbor, Michigan; ${ }^{6}$ Nationwide Children's Hospital and The Center for Childhood Cancer at The Research Institute, Columbus, Ohio

Conflict of interest: Nothing to Report.

Grant sponsor: Sarcoma Alliance for Research.

*Correspondence to: E. Anders Kolb, Division of Hematology/ Oncology, Alfred I. duPont Hospital for Children, 1600 Rockland Road, Wilmington, DE 19803. E-mail: eakolb@nemours.org

Received 7 December 2009; Accepted 21 January 2010 
monoclonal antibodies capable of inhibiting the binding of IGF-1 and IGF-2, and small molecules capable of inhibiting signaling through IGF-1R without excessive toxicity, has generated renewed interest in defining the validity of this target and biomarkers for response. There are currently at least six human or fully humanized anti-IGF-1R monoclonal antibodies currently in clinical trials, each binding to the IGF-1R receptor and inhibiting ligand binding. There are as well small molecules that target IGF-1R kinase domain. However, markers for activity and resistance to IGF-1R inhibitors in osteosarcoma are neither known, nor is there an understanding of the cellular events that take place following IGF-1R inhibition in osteosarcoma. To utilize potent inhibitors of IGF-1R signaling effectively, it is important to understand the specific cellular events that predict sensitivity or offer a means for tumors to salvage effective growth signaling in the absence of IGF-1R activation. In this current report, the fully human monoclonal antibody R1507 is evaluated in a panel of pediatric osteosarcoma tumor lines, both alone and in combination with mTor inhibitor rapamycin. As previously reported in other sarcomas, rapamycin increases signaling through AKT in a manner dependent on IGF-1R [9]. R1507 inhibits this activation in all tumor lines evaluated and improves response in two of the lines.

\section{METHODS}

\section{Tumor Lines and Reagents}

The OS1, OS2, OS9, OS17, OS31, and OS33 human osteosarcoma tumor lines were generously provided by the Pediatric Preclinical Testing Program. These lines are maintained by serial passage in severe combined immune deficient (SCID) mice as described previously by the Pediatric Preclinical Testing Program [10].

R1507 was provided by Roche Diagnostics GmbH (Discovery Oncology, Penzberg, Germany). R1507 was at a stock concentration of $25.6 \mathrm{mg} / \mathrm{ml}$. For administration to the mice, the stock solution was diluted in sterile saline and administered weekly for 6 weeks at a dose of $10 \mathrm{mg} / \mathrm{kg}$ via intraperitoneal injection. Rapamycin was purchased from LC Laboratories (Woburn, MA) and dissolved in DMSO to a 5\% solution. Rapamycin was further diluted in 5\% Tween 80 in water and administered to mice via intraperitoneal injection daily five times for six consecutive weeks at a dose of $5 \mathrm{mg} / \mathrm{kg} /$ day.

\section{Immunoblotting}

At specified time points, mice were sacrificed with $\mathrm{CO}_{2}$, and tumors immediately harvested on ice. The tumors were then lysed in RIPA with buffer complete protease inhibitor cocktail $G$ and phosphatase inhibitor cocktail 1 and 2 (Sigma, St. Louis, MO). The protein concentrations of the tumor lysates were determined by the BCA protein assay (Pierce, Rockford, IL). Twenty micrograms of protein from the lysates was resolved by SDS-PAGE and transferred to nitrocellulose membranes. Membranes were blocked with 5\% milk in TBS-Tween 20 and then incubated with various primary antibodies overnight at $4{ }^{\circ} \mathrm{C}$. HRP-conjugated anti-rabbit secondary antibody (Cell Signaling Technology, Cambridge, MA) was used to detect protein-bound primary antibody. Bound secondary antibody was detected by using Amersham ECL Plus western blotting detection reagents (GE Healthcare, Piscataway, NJ). The blots were then exposed to a Kodak Biomax Light Film.
For immunoblotting, antibodies against AKT, phospho-AKT (Ser473), IGF-1R beta, MAPK p44/p42, phospho-p44/42 MAPK (Thr202/Tyr204), S6 ribosomal protein, and phospho-S6 ribosomal protein (Cell Signaling Technology) were used.

\section{Animal Studies}

Five- to 6-week-old female CB17 SCID mice (Taconic Farms, Germantown, NY) were used for the in vivo experiments using methodology previously described [10]. When implanted xenograft tumors reached a volume between 200 and $500 \mathrm{~mm}^{3}$, the mice bearing tumors were randomized into treatment groups consisting of 8-10 mice. All mice were maintained under barrier conditions, and experiments were conducted using protocols and conditions approved by the institutional animal care and use committee of the Albert Einstein College of Medicine of Yeshiva University.

Tumor growth was assessed weekly for the duration of the study initiation (6 weeks unless otherwise specified). Tumor volumes were assumed to be spherical, and calculated from the formula $(\pi / 6) \times d^{3}$, where $d$ represents the mean diameter. Responses were determined using tumor volume treatment/control (T/C), event free survival T/C, and objective response criteria as defined previously [10]. An event is defined as (1) death, (2) a reduction in starting body weight of $20 \%$, or (3) a relative tumor volume (RTV) greater than or equal to 4 , where the RTV is the volume on the day of measurement divided by the volume on day 0 of treatment (starting volumes were $0.2-0.3 \mathrm{~cm}^{3}$ ). Animals reaching an RTV of 4 prior to the completion of the 6-week experiment were euthanized and the event was recorded.

Using criteria defined previously by Houghton et al. [10], progressive disease is defined as less than $50 \%$ regression from original tumor volume for the entire study period (RTV $>0.5)$ and greater than $25 \%$ increase in tumor volume at the end of the study period $(\mathrm{RTV}>1.25)$. Stable disease is defined as tumor regression that did not exceed $50 \%$ of the original tumor volume throughout the entire study period (RTV $>0.5)$ and less than $25 \%$ increase in tumor volume at the end of the study period $(\mathrm{RTV}<1.25)$. A partial response is defined as greater than $50 \%$ regression in tumor volume $(\mathrm{RTV}<0.5)$ but with a measurable tumor mass of greater than $0.10 \mathrm{~cm}^{3}$. Loss of measurable tumor mass $\left(<0.10 \mathrm{~cm}^{3}\right)$ at any point during the treatment period ( 6 weeks) was defined as a complete response (CR). A maintained $\mathrm{CR}$ was defined as a loss of measurable tumor mass $\left(<0.10 \mathrm{~cm}^{3}\right)$ at any point after initiation of therapy without re-growth during the 6-week study period.

\section{Statistical Methods}

RTV values were compared with the nonparametric Wilcoxon rank sum test with Bonferroni's correction. The survival analysis for each treatment was determined using the Kaplan-Meier method. Pairwise comparisons of the Kaplan-Meier curves were performed by a log-rank test with Bonferroni's correction. The difference was considered to be significant when the $P$ value was less than 0.05 .

\section{RESULTS \\ R1507 Inhibits Growth of Human Osteosarcoma Xenografts}

Tumor responses to R1507 treatment are presented in Figure 1. The OS1, OS2, OS9, and OS17 tumors all had tumor growth delays 
a OS2

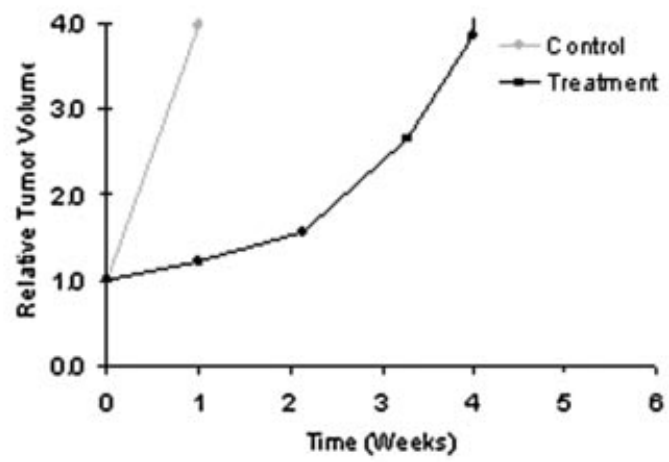

c os 31

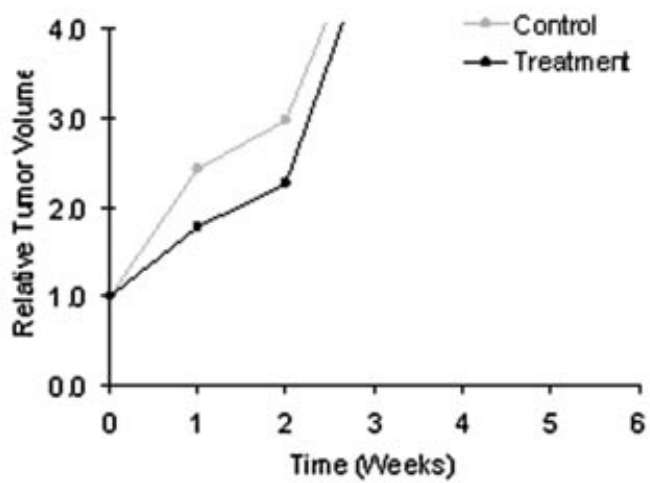

e
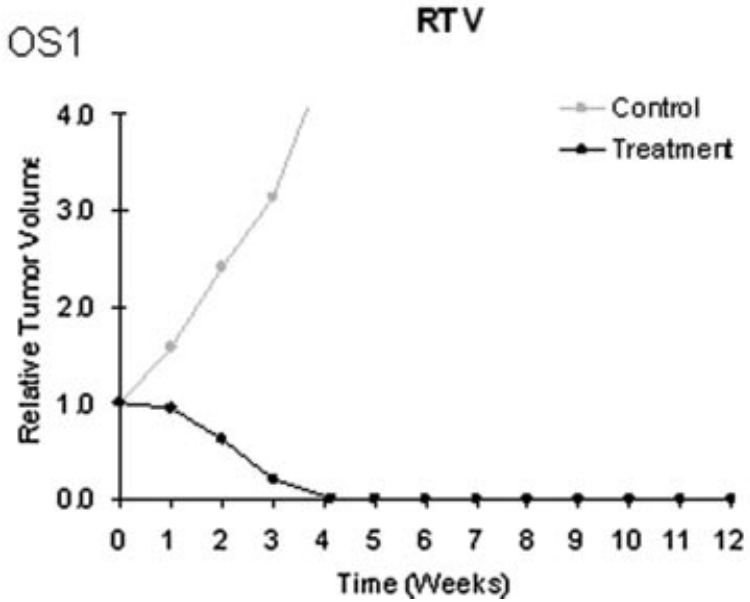

b OSg

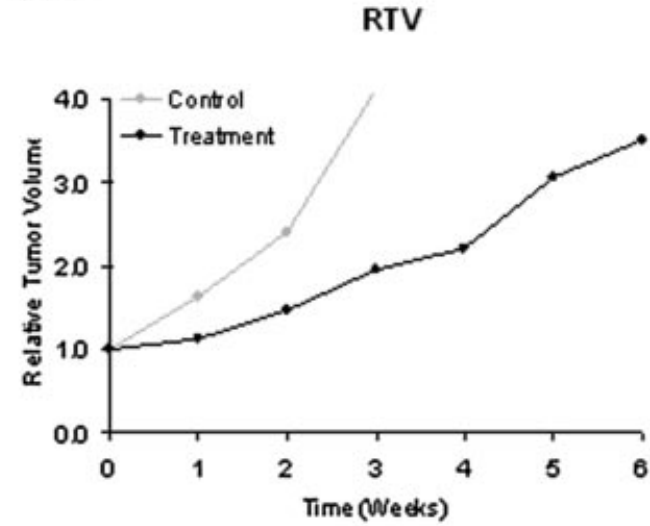

d 0 os 33

RTV

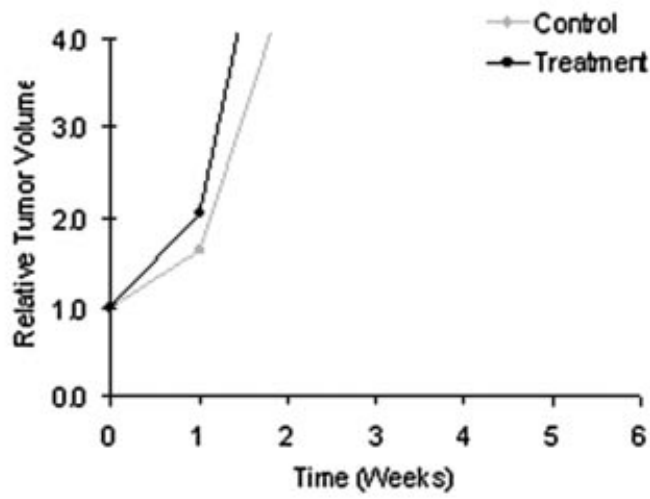

f OS17 RTV

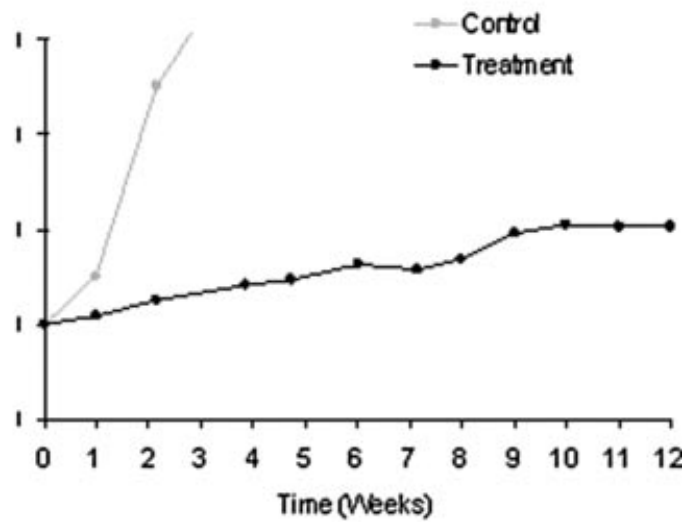

Fig. 1. Xenograft growth curves for osteosarcoma xenografts treated with R1507. The relative tumor volume (RTV) is calculated as the ratio of tumor volume on the day of measurement divided by the tumor volume on day 0 . Mice were treated with R $1507 \mathrm{weekly}$ at a dose of $10 \mathrm{mg} / \mathrm{kg}$ for a total of 6 weeks. For OS2 (a), OS9 (b), OS31 (c), and OS33 (d), the experiment ended at the completion of the 6-week treatment period. OS1 (e) and OS17 (f) were observed for a total of 12 weeks without additional treatment after week 6 . Control-treated mice received only vehicle on same schedule. Statistical analysis is listed in Table I.

with statistically significant improvements in event-free survival and day 22 median tumor volumes (Table I). There was minimal change in tumor growth in the OS31 and OS33 tumors with no effect on event-free survival. These results are consistent with the previously published reports with other IGF-1R directed antibodies in the same tumor panel $[11,12]$.

Treatment of OS1 and OS17 with R1507 for 6 weeks and observation for an additional 6 weeks are shown in Figure 1e,f. In 
TABLE I. Summary of Tumor Response to R1507

\begin{tabular}{|c|c|c|c|c|c|}
\hline \multirow{2}{*}{$\frac{\text { Tumor }}{\text { OS1 }}$} & \multicolumn{2}{|c|}{$\begin{array}{l}\text { Kaplan-Meier estimate of } \\
\text { median time to event }\end{array}$} & \multirow{2}{*}{$\begin{array}{l}P \text { value } \\
<0.0001\end{array}$} & \multirow{2}{*}{$\begin{array}{c}\begin{array}{c}\text { Day } 22 \text { tumor volume } \\
\text { (treated/control) }\end{array} \\
0.06\end{array}$} & \multirow{2}{*}{$\begin{array}{l}P \text { value } \\
<0.0001\end{array}$} \\
\hline & $\begin{array}{l}\text { Control } \\
\text { Treated }\end{array}$ & $\begin{array}{c}36 \text { days } \\
>\mathrm{EP}^{\mathrm{b}}\end{array}$ & & & \\
\hline OS2 & $\begin{array}{l}\text { Control } \\
\text { Treated }\end{array}$ & $\begin{array}{l}15 \text { days } \\
29 \text { days }\end{array}$ & $<0.0001$ & 0.20 & $<0.0001$ \\
\hline OS9 & $\begin{array}{l}\text { Control } \\
\text { Treated }\end{array}$ & $\begin{array}{l}22 \text { days } \\
>\mathrm{EP}\end{array}$ & $<0.0001$ & 0.31 & $<0.0001$ \\
\hline $\operatorname{OS} 17^{\mathrm{a}}$ & $\begin{array}{l}\text { Control } \\
\text { Treated }\end{array}$ & $\begin{array}{c}22 \text { days } \\
>\mathrm{EP}\end{array}$ & $<0.0001$ & 0.40 & $<0.0001$ \\
\hline OS31 & $\begin{array}{l}\text { Control } \\
\text { Treated }\end{array}$ & $\begin{array}{l}22 \text { days } \\
22 \text { days }\end{array}$ & 0.95 & 1.02 & 1.00 \\
\hline OS33 & $\begin{array}{l}\text { Control } \\
\text { Treated }\end{array}$ & $\begin{array}{l}15 \text { days } \\
15 \text { days }\end{array}$ & 0.24 & 0.93 & 0.68 \\
\hline
\end{tabular}

${ }^{a}$ Animals observed for a total of 12 weeks following a total of 6 weeks of treatment; ${ }^{b} \mathrm{EP}$ is experimental period. For OS2, OD9, OD31, and OS33, the tumors were followed for the 6 weeks of treatment. For OS1 and OS17, the tumors were followed for an additional 6 weeks (12 weeks total) to observe the duration of response.

OS1, the CR was maintained for 6 weeks after R1507was discontinued. Three of the ten OS1-treated mice had palpable tumors (all less than $0.05 \mathrm{~cm}^{3}$, and still meeting the criteria for a CR). During weeks 6-12 of the experiment, at which time no R1507 was administered, these tumors continued to grow, albeit at a slower rate than controls. Likewise, the slope of the curve for OS17 did not change when R1507 therapy was discontinued. There was clearly tumor growth, but at a slower rate than the controls. This inhibition of growth rate persisted 6 weeks after the last treatment. There were no drug-related toxicities noted in the treated mice.

\section{IGF-1R Expression Does Not Predict Sensitivity to IGF-1R Inhibition in Osteosarcoma}

IGF-1R expression in the osteosarcoma xenograft lines as visualized by immunoblotting is presented in Figure 2. These data

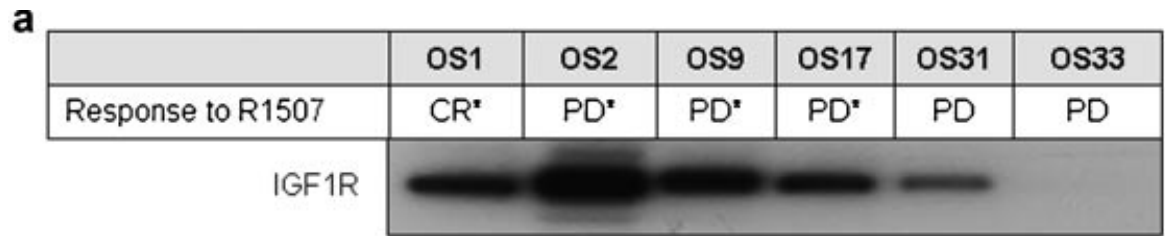

b
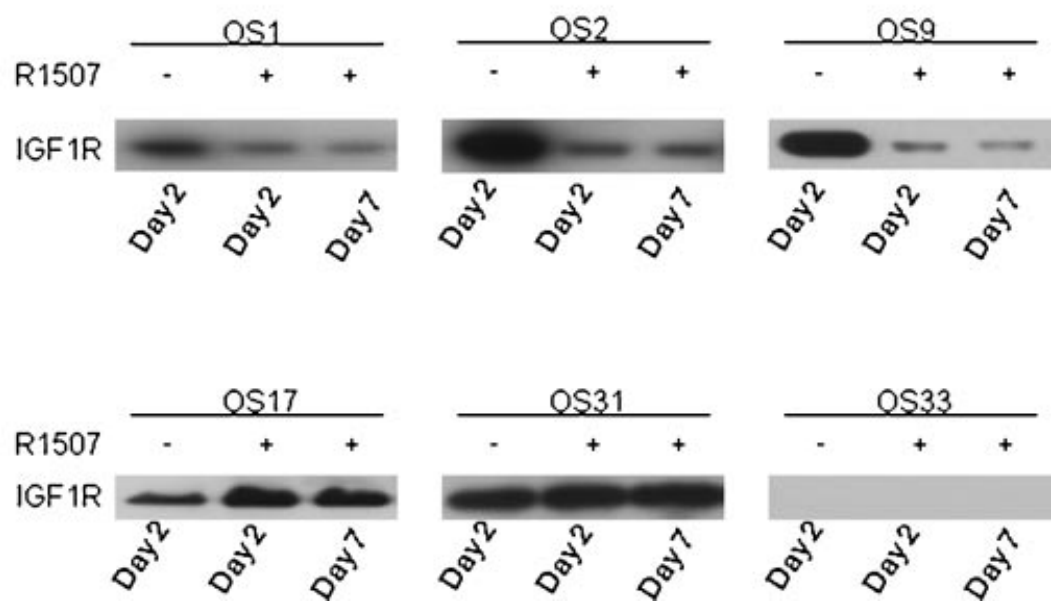

Fig. 2. Determination of IGF-1R expression in xenograft tumors treated with R1507. a: The maximal response to R1507 is depicted in the table, along with the corresponding constitutive IGF-1R expression. These immunoblots represent constitutive expression of IGFR in untreated tumors at volume of $0.5 \mathrm{~cm}^{3}$. CR, complete response; PD, progressive disease. *Response where there was a statistically significant improvement in event-free survival. b: Mice received either vehicle or a single dose of R1507 (10 mg/kg) on day 0. Tumors were harvested and snap frozen on days 2 and 7. Immunoblotting for total IGF-1R was performed. 
a os1

a. OS1

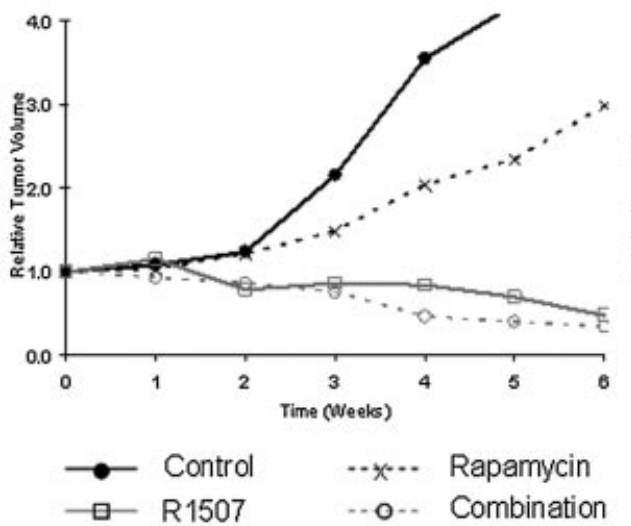

c OS9
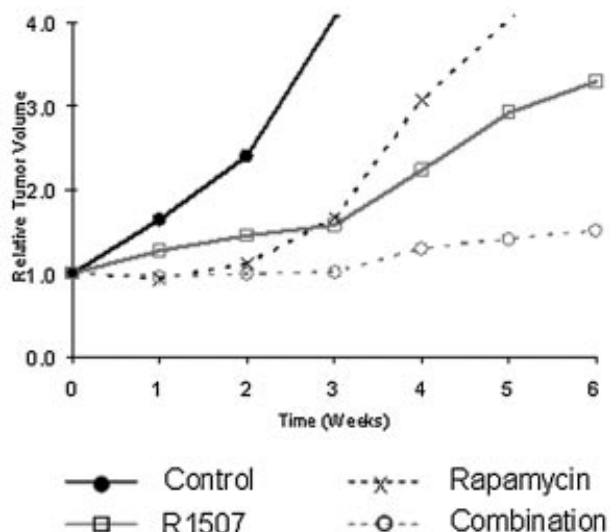

e $0 \$ 31$

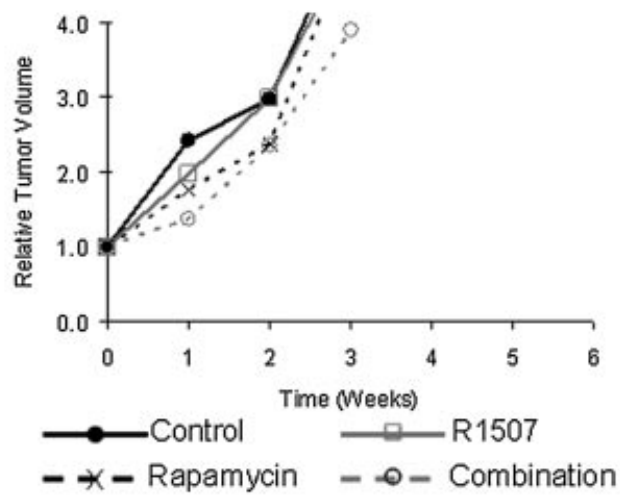

b $\quad$ OS2

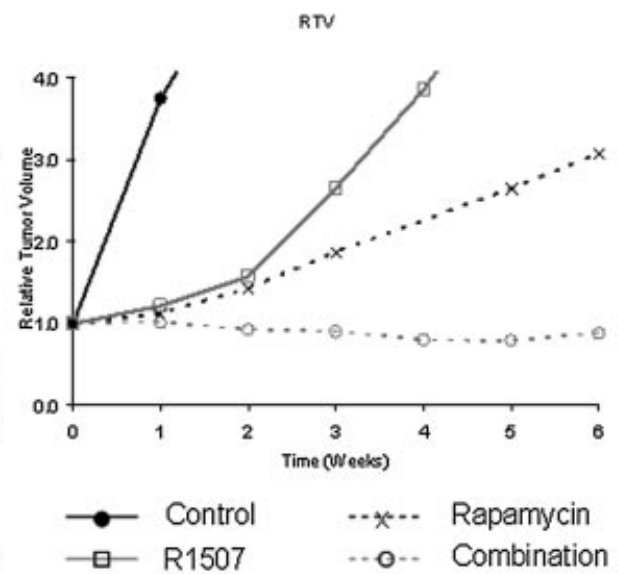

d $\quad$ OS17 RTV
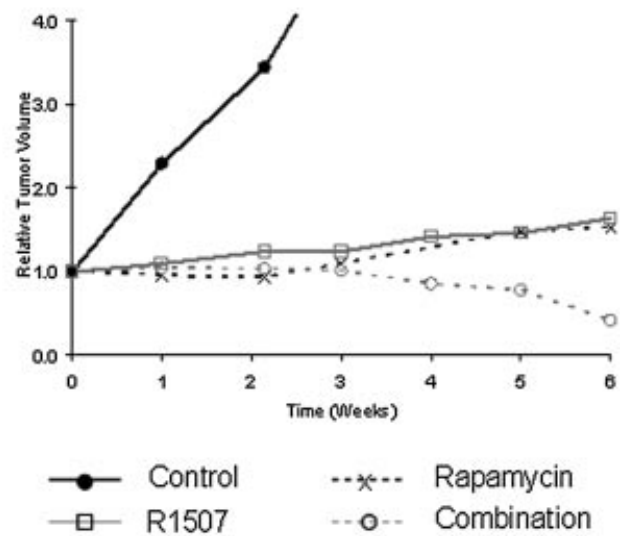

RTV

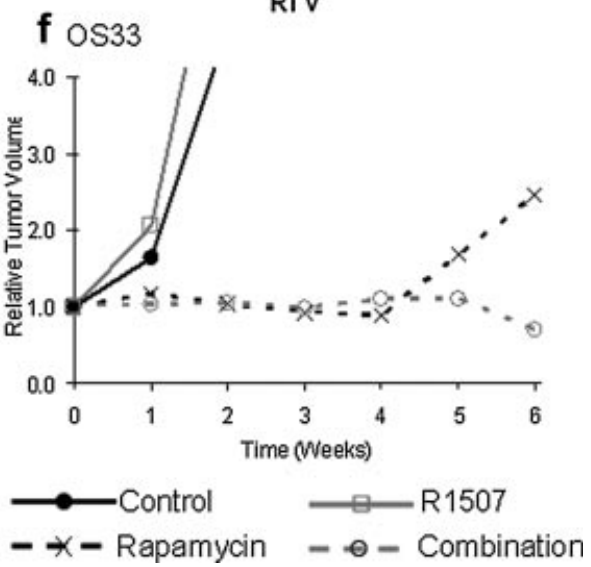

Fig. 3. Determination of osteosarcoma xenograft response to R1507 in combination with rapamycin. Tumor-bearing mice were randomized into one of four treatment groups and received either vehicle R $150710 \mathrm{mg} / \mathrm{kg} /$ week IP for 6 weeks, rapamycin $5 \mathrm{mg} / \mathrm{kg} / \mathrm{day}$ IP 5 days per week for 6 weeks or the combination of rapamycin and R1507. Each data point is the median of 8-10 mice per treatment group. Statistical analysis is detailed in Table II. 
correlate to the publicly available RNA expression (data not shown) using the Affymetrix U133 Plus 2.0 chip $[11,13]$. OS33 has low level expression of IGF-1R and is insensitive to all the three antibodies. However, in the four R1507 sensitive lines, expression did not correlate with response. The OS1 tumor was most sensitive to R1507 with a CR. Compared to the other xenograft tumors, OS1 had intermediate receptor expression. OS2 had the highest level of expression by immunoblotting, and yet demonstrates progressive disease in response to R1507 treatment.

A decrease in IGF-1R expression was seen in three of the four R1507 sensitive cell lines (OS1, OS2, OS9, but not OS17) with R1507 therapy.

\section{Following Inhibition of mTor, Osteosarcoma Tumor Lines Induce Alternate Pathways to Salvage Oncogenic Growth Signaling}

In data published previously, the Pediatric Preclinical Testing Program demonstrated that rapamycin (a specific inhibitor of the mTOR/raptor complex) inhibits growth of the same xenografts presented in this report [14]. It has also been previously reported in sarcomas that rapamycin may induce phosphorylation of AKT in a manner dependent on IGF-1R, thus increasing the dependency of the tumor on IGF-1R mediated signaling [15-17]. Figure 3 shows the results of the rapamycin in combination with R1507 in the OS xenograft lines. In OS2, OS9, and OS31, there was an increase in activity of the combination when compared to either drug alone (Table II). In each of these lines, the day 22 RTV in the combination arm is significantly smaller than the other treatment arms. While not demonstrating a difference at day 22, in OS17 on day 42, the RTV in the combination is significantly smaller than the other treatment arms. In the OS1 tumor lines, there is a CR with R1507 alone, and no additional effect of the combination. In OS33, there seems to be no enhanced activity with the combination at day 22 . There were no drug-related toxicities noted in the treated mice.

Figure 4 demonstrates that rapamycin does induce phosphorylation of AKT in all of the xenograft tumor lines. When R1507 was added to the rapamycin therapy, signaling through both AKT and ribosomal S6 kinase (as a marker of mTOR activation) was inhibited.

\section{Signaling Through the Ras/MAPK Pathway May Predict Susceptibility of Osteosarcoma Cells to IGF-1R Signaling}

In the OS31 and OS33 xenograft tumor lines, neither of which has demonstrated a response to R1507, there was an increase in MAPK phosphorylation on day 2 and 7 after a single treatment with the IGF-1R antibody (Fig. 4e,f). These data imply that signaling through MAPK may be induced when signaling though IGF-1R is inhibited.

The increase in MAPK phosphorylation in response to R1507 may be dependent on signaling through mTOR. On day 2 and 7 of treatment with rapamycin alone, MAPK phosphorylation was diminished in all tumor lines. On day 2 of the combination treatment, there was partial decrease in MAPK phosphorylation in OS31, and a significant decrease in OS33.

In the four tumor lines sensitive to R1507, there is either a decrease or no change in MAPK phosphorylation. In the OS1 tumor line, there was complete tumor regression following administration

TABLE II. Summary of Tumor Response to R1507 and Rapamycin

\begin{tabular}{|c|c|c|c|c|c|}
\hline \multirow{2}{*}{$\frac{\text { Tumor }}{\mathrm{OS} 1^{\mathrm{a}}}$} & \multicolumn{2}{|c|}{$\begin{array}{l}\text { Kaplan-Meier estimate of } \\
\text { median time to event }\end{array}$} & \multirow{2}{*}{$\frac{P \text { value }^{\mathrm{a}}}{<0.0001}$} & \multirow[t]{2}{*}{$\begin{array}{l}\text { Day } 22 \text { tumor volume } \\
\text { (treated/control) }\end{array}$} & \multirow[t]{2}{*}{$P$ value $^{\mathrm{a}}$} \\
\hline & Control & 36 days & & & \\
\hline & Rapamycin & $>\mathrm{EP}$ & 0.20 & 0.73 & 0.001 \\
\hline & R1507 & $>\mathrm{EP}$ & 1.0 & 0.40 & 1.00 \\
\hline & Combination & $>\mathrm{EP}$ & & 0.35 & \\
\hline \multirow[t]{4}{*}{ OS2 } & Control & 15 days & $<0.0001$ & & $<0.0001$ \\
\hline & Rapamycin & $>\mathrm{EP}$ & 0.63 & 0.14 & $<0.0001$ \\
\hline & $\mathrm{R} 1507$ & 32 & $<0.0001$ & 0.20 & $<0.0001$ \\
\hline & Combination & $>\mathrm{EP}$ & & 0.07 & \\
\hline \multirow[t]{4}{*}{ OS9 } & Control & 22 days & $<0.0001$ & & $<0.0001$ \\
\hline & Rapamycin & 38.5 days & 0.002 & 0.39 & 0.017 \\
\hline & $\mathrm{R} 1507$ & $>\mathrm{EP}$ & 1.0 & 0.37 & 0.003 \\
\hline & Combination & $>\mathrm{EP}$ & & 0.24 & \\
\hline \multirow[t]{4}{*}{$\mathrm{OS} 17^{\mathrm{a}}$} & Control & 22 days & $<0.0001$ & & $<0.0001$ \\
\hline & Rapamycin & $>\mathrm{EP}$ & 1.00 & 0.21 & 1.00 \\
\hline & $\mathrm{R} 1507$ & $>\mathrm{EP}$ & 1.00 & 0.24 & 0.84 \\
\hline & Combination & $>\mathrm{EP}$ & & 0.18 & \\
\hline \multirow[t]{4}{*}{ OS31 } & Control & 22 days & 0.002 & & 0.002 \\
\hline & Rapamycin & 22 days & 0.066 & 0.76 & 0.009 \\
\hline & $\mathrm{R} 1507$ & 22 days & 0.006 & 0.97 & 0.002 \\
\hline & Combination & 29 days & & 0.58 & \\
\hline \multirow[t]{4}{*}{ OS33 } & Control & 14 days & $<0.0001$ & & $<0.0001$ \\
\hline & Rapamycin & $>\mathrm{EP}$ & 1.0 & 0.18 & 1.0 \\
\hline & $\mathrm{R} 1507$ & 15 days & $<0.0001$ & 0.27 & $<0.0001$ \\
\hline & Combination & 29 days & & 0.018 & \\
\hline
\end{tabular}

EP, experimental period, 6 weeks for all experiments. ${ }^{\text {a }} P$-value of the combination group compared control, rapamycin or R1507 groups. 

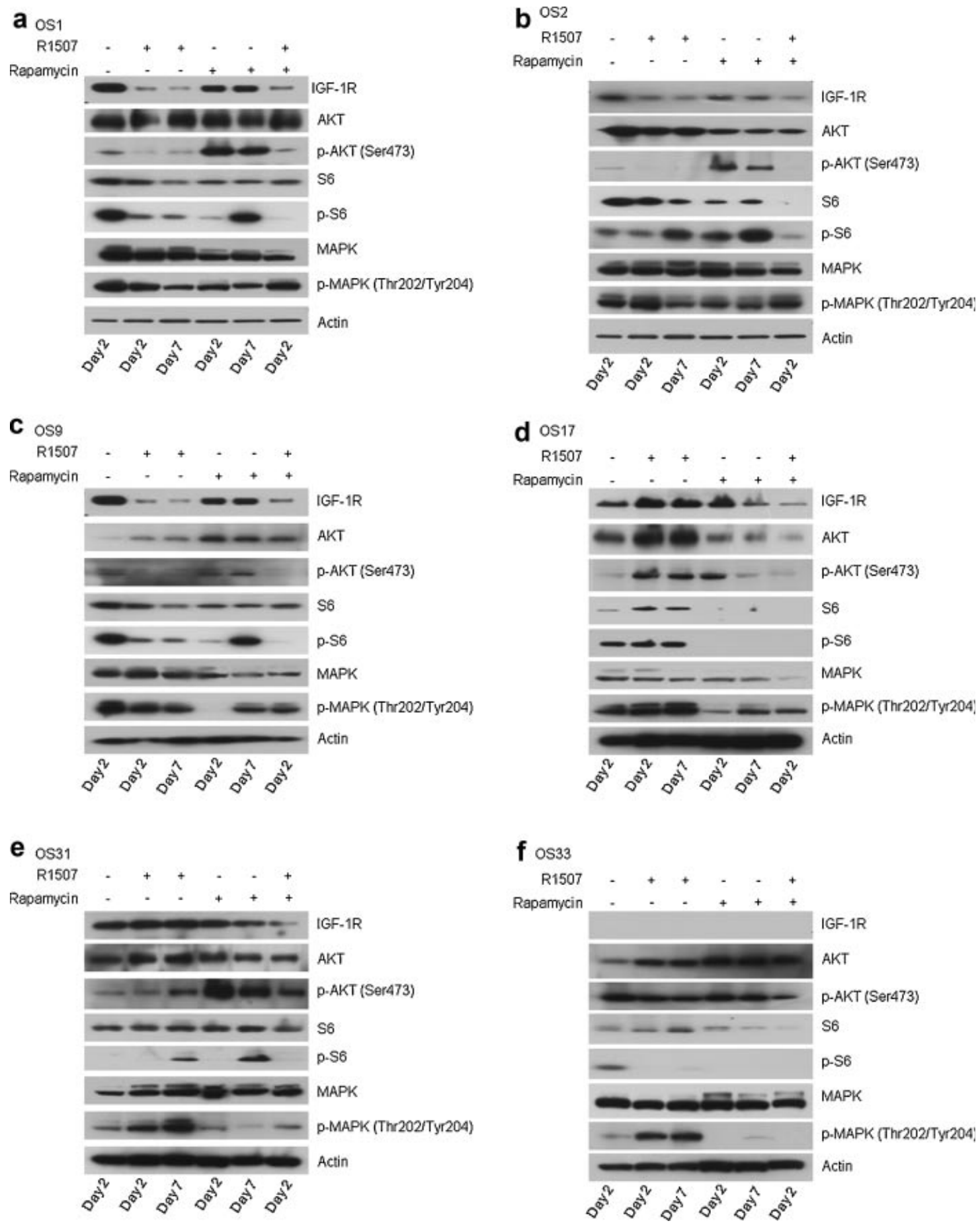

Fig. 4. Correlation of signaling through AKT and mTor with response in tumor treated with R1507 and rapamycin. Tumors were harvested and snap frozen from three mice in each treatment group as in Figure 3 on days 0,2, and 7. All immunoblots were performed in triplicate and representative results are shown. Immunoblots for OS 1 (a), OS 2 (b), OS9 (c), OS17 (d), OS31 (e), and OS33 (f) are presented.

of the R1507 anti-IGF-1R antibody. Seven days after treatment with R1507 alone, there was inhibition of AKT, ribosomal S6 kinase and MAPK phosphorylation at Thr202/Tyr204 (Fig. 4a). In the OS2 and OS9 tumor lines, there was also a decrease in AKT phosphorylation, at least a transient decrease in S6 kinase phosphorylation, and a decrease, in MAPK phosphorylation (Fig. 4b,c). In OS17, there was no change in MAPK phosphorylation with R1507.

\section{DISCUSSION}

In six well-characterized osteosarcoma xenograft tumor lines, R1507 has anti-tumor activity consistent with the published results of two other monoclonal antibodies targeting IGF-1R [11,12]. For each of the three anti-IGF-1R antibodies we have evaluated, there is anti-tumor activity in the OS1, OS2, OS9 and OS17 tumors, 
and minimal activity in OS31 and SO33. It has been reported in Ewing sarcoma and rhabdomyosarcoma that inhibition of mTOR may increase the dependency of tumors on IGF signaling [9,15,17]. Kurmasheva et al. [9]. recently showed that inhibitors of IGF-1R act synergistically with rapamycin in sarcoma xenografts by inhibiting hyperphosphorylation of AKT in response to mTOR inhibition. In the current report, R1507 inhibits the induction of AKT phosphorylation by rapamycin, and in three of the six tumor lines there is enhanced anti-tumor effect.

R1507 induces statistically significant improvements in eventfree survival in OS1, OS2, OS9, and OS17, including a CR in OS1. In the R1507-sensitive tumors, OS1, OS2, and OS9 phosphorylation of AKT and S6 kinase are inhibited by R1507. In the OS17 tumor, total AKT and total S6 ribosomal protein, as well as phospho AKT and phospho S6 kinase are increased with R1507 treatment. The mechanism of the anti-tumor effect of R1507 in this tumor is being further evaluated.

We demonstrate that in the two R1507-resistant xenograft tumor lines, phosphorylation of MAPK is induced within 7 days of a single dose of antibody. Interestingly, this effect seems to be dependent on signaling through mTOR, as evidenced by the fact that R1507induced MAPK phosphorylation is inhibited by rapamycin. In the sensitive tumor lines, constitutive MAPK phosphorylation is decreased (OS1, OS2, and OS9) or remains the same (OS17) with R1507 treatment. Rapamycin has been shown to partially suppress p44/42 MAPK activation by serum in melanoma cells [18]. Harwood et al. [19] recently demonstrated that the mTOR-raptor complex (mTORC1) has a complex regulatory role in MAPK signaling. In rhabdomyosarcoma lines, mTORC1 modulates phosphorylation of MAPK at Thr202 through protein phosphatase 2A [19]. The mechanism by which MAPK phosphorylation is increased by R1507 in osteosarcoma tumors consistently resistant to IGF-1R inhibitors is currently under investigation.

In the osteosarcoma tumor lines evaluated in this report, IGF-1R expression does not predict response to R1507. These data are not consistent with the report by Cao et al. [16]. who recently showed, in rhabdomyosarcoma tumors, that sensitivity to anti-IGF-1R antibody therapy corresponds directly to IGF-1R receptor level. In osteosarcoma, there may be more robust markers for response than receptor expression. Illustrating this point is the fact that the OS1 tumor demonstrated a CR to R1507 but with intermediate receptor expression. The OS2 tumor has high receptor expression with progressive disease in response to R1507, albeit with an improvement in event-free-survival. The R1507-resistant tumors, OS31, and OS33 were found to have low level and no IGF-1R expression, respectively, by western blotting. However, despite the low receptor expression, there is still evidence of an effect on signaling illustrated through an induction of MAPK phosphorylation. Conclusions about relative expression of IGF-1R using comparative immunoblotting are limited and not as reliable as the receptor quantification methodology utilized by Cao et al. Nonetheless, given the data presented above, it would appear that alternate signaling through MAPK may be more informative of IGF-1R resistance in these osteosarcoma xenograft tumors. Our work demonstrates that IGF$1 \mathrm{R}$ expression is necessary but not sufficient to predict response to IGF-1R inhibition. Activation of alternative proliferation and survival pathways including MAPK may play a role in resistance to IGF-1R inhibition.

The OS1 and OS17 tumors were observed for 6 weeks following treatment with R1507. In neither of these tumors was there an increase in tumor growth rate following discontinuation of the drug. It is reasonable to hypothesize that under the influence of R1507, the cells invoke an alternative signaling pathway and that this pathway maintains the oncogenic growth signals. In OS17, growth is permitted, but is apparently less efficient. Furthermore, it is possible, given these data, that the salvage signaling changes induced by IGF-1R inhibition are long-standing or even permanent, suggesting altered expression of signaling pathway mediators. These hypotheses are currently under investigation.

The significance of identifying markers that will predict whether a tumor is sensitive or resistant to IGF-1R-directed therapy cannot be overstated. Clearly, in Figure 1, there is a subset of osteosarcoma tumors that is sensitive to IGF-1R-directed therapy and a subset that is resistant, regardless of receptor expression. In rhabdomyosarcoma cell lines, treatment with rapamycin induces phosphorylation of AKT. mTOR inhibits cap-dependent translation and, in doing so, has the potential to inhibit expression of receptor tyrosine kinases PI3K and AKT [15]. Releasing this negative feedback signal with rapamycin has the potential to increase signaling through AKT allowing increased expression and activation of upstream signal mediators or AKT itself. AKT may also be directly phosphorylated through the mTOR/rictor complex, which is less susceptible to inhibition by rapamycin. In osteosarcoma, the current report demonstrates that this increase in AKT phosphorylation is dependent on IGF-1R signaling. Further studies are needed to confirm whether it is possible for IGF-1R-independent signaling through Ras to provide a means for osteosarcoma to salvage growth signaling in the presence of IGF-1R signal inhibition. Increased signaling through the Ras/MAPK pathway may be mediated though an activating mutation within the components of the Ras pathway or through alterations in upstream signaling pathways.

\section{ACKNOWLEDGMENT}

This research work was supported in part by a grant from the Sarcoma Alliance for Research through Collaboration.

\section{REFERENCES}

1. Meyers PAGR. Osteosarcoma. Pediatr Clin North Am 1997;44: 973-989.

2. McCarthy TL, Centrella M. Local IGF-I expression and bone formation. Growth Horm IGF Res 2001;11:213-219.

3. Burrow S, Andrulis IL, Pollak M, et al. Expression of insulin-like growth factor receptor, IGF-1, and IGF-2 in primary and metastatic osteosarcoma. J Surg Oncol 1998;69:21-27.

4. Pollak M, Sem AW, Richard M, et al. Inhibition of metastatic behavior of murine osteosarcoma by hypophysectomy. J Natl Cancer Inst 1992;84:966-971.

5. Savage SA, Woodson K, Walk E, et al. Analysis of genes critical for growth regulation identifies insulin-like growth factor 2 receptor variations with possible functional significance as risk factors for osteosarcoma. Cancer Epidemiol Biomarkers Prev 2007; 16:16671674.

6. Hermanto U, Zong CS, Wang LH. Inhibition of mitogen-activated protein kinase kinase selectively inhibits cell proliferation in human breast cancer cells displaying enhanced insulin-like growth factor I-mediated mitogen-activated protein kinase activation. Cell Growth Differ 2000;11:655-664.

7. Kurmasheva RT, Houghton PJ. IGF-I mediated survival pathways in normal and malignant cells. Biochim Biophys Acta 2006;1766: $1-22$. 
8. El-Badry OM, Minniti C, Kohn EC, et al. Insulin-like growth factor II acts as an autocrine growth and motility factor in human rhabdomyosarcoma tumors. Cell Growth Differ 1990;1:325331.

9. Kurmasheva RT, Dudkin L, Billups C, et al. The insulin-like growth factor-1 receptor-targeting antibody, CP-751,871, suppresses tumor-derived VEGF and synergizes with rapamycin in models of childhood sarcoma. Cancer Res 2009;69:76627671.

10. Houghton PJ, Morton CL, Tucker C, et al. The pediatric preclinical testing program: Description of models and early testing results. Pediatr Blood Cancer 2007;49:928-940.

11. Kolb EAGR, Houghton PJ, Morton CL, et al. Initial testing (stage 1) of a monoclonal antibody (SCH 717454) against the IGF-1 receptor by the pediatric preclinical testing program. Pediatr Blood Cancer 2008;50:1190-1197.

12. Kolb EAMCL, Houghton PJ, Maris JM, et al. Pediatric preclinical testing program (PPTP) evaluation of the fully human anti-IGF-1R antibody IMC-A12. Eur J Cancer 2008;6: 176.

13. PPTP, Affy Data. 2008; [cited]; Available from: http://pptp.stjude. org/affyData.php.
14. Houghton PJ, Morton CL, Kolb EA, et al. Initial testing (stage 1) of the mTOR inhibitor rapamycin by the pediatric preclinical testing program. Pediatr Blood Cancer 2008;50:799-805.

15. Wan X, Harkavy B, Shen N, et al. Rapamycin induces feedback activation of AKT signaling through an IGF-1R-dependent mechanism. Oncogene 2007;26:1932-1940.

16. Cao LYY, Darko I, Currier D, et al. Addiction to elevated insulinlike growth factor I receptor and initial modulation of the AKT pathway define the responsiveness of rhabdomyosarcoma to the targeting antibody. Cancer Res 2008;68:8039-8048.

17. Kurmasheva RBC, Phelps D, Morton C, et al. Human monoclonal antibody CP751871, combined with rapamycin is effective treatment for childhood sarcoma xenografts. AACR Meeting Abstracts, Oct 2007; 2007: C172 2008.

18. Molhoek KR, Brautigan DL, Slingluff CL, Jr. Synergistic inhibition of human melanoma proliferation by combination treatment with B-Raf inhibitor BAY 43-9006 and mTOR inhibitor rapamycin. J Transl Med 2005;3:39.

19. Harwood FC, Shu L, Houghton PJ. mTORC1 signaling can regulate growth factor activation of $\mathrm{p} 44 / 42$ mitogen-activated protein kinases through protein phosphatase 2A. J Biol Chem 2008;283:2575-2585. 